\title{
Carbon Source Affects Synthesis, Structures, and Activities of Mycelial Polysaccharides from Medicinal Fungus Inonotus obliquus
}

\author{
Huihui He, Yingying Li, Mingyue Fang, Tiantian Li, Yunxiang Liang, and Yuxia Mei* \\ State Key Laboratory of Agricultural Microbiology, College of Life Science and Technology, Huazhong Agricultural
} University, Wuhan 430070, P.R. China

\begin{abstract}
The effects of various carbon sources on mycelial growth and polysaccharide synthesis of the medicinal fungus Inonotus obliquus in liquid fermentation were investigated. After 12-d fermentation, mycelial biomass, polysaccharide yield, and polysaccharide content were significantly higher in Glc+Lac group (glucose and lactose used as combined carbon source) than in other groups. Crude polysaccharides (CIOPs) and the derivative neutral polysaccharides (NIOPs) were obtained from mycelia fermented using GIc, fructose (Fru), Lac, or Glc+Lac as carbon source. Molecular weights of four NIOPs (termed as NIOPG, NIOPF, NIOPL, and NIOPGL) were respectively $780.90,1105.00,25.32$, and $10.28 \mathrm{kDa}$. Monosaccharide composition analyses revealed that NIOPs were composed of GIc, Man, and Gal at different molar ratios. The NIOPs were classified as a-type heteropolysaccharides with $1 \rightarrow 2,1 \rightarrow 3,1 \rightarrow 4,1 \rightarrow 6$ linkages in differing proportions. In in vitro cell proliferation assays, viability of RAW264.7 macrophages was more strongly enhanced by NIOPL or NIOPGL than by NIOPG or NIOPF, and proliferation of HeLa or S180 tumor cells was more strongly inhibited by NIOPG or NIOPGL than by NIOPF or NIOPL, indicating that immune-enhancing and antitumor activities of NIOPs were substantially affected by carbon source. qRT-PCR analysis revealed that expression levels of phosphoglucose isomerase (PGI) and UDP-GIc 4-epimerase (UGE), two key genes involved in polysaccharide synthesis, varied depending on carbon source. Our findings, taken together, clearly demonstrate that carbon source plays an essential role in determining structure and activities of $l$. obliquus polysaccharides by regulating expression of key genes in polysaccharide biosynthetic pathway.
\end{abstract}

Keywords: Inonotus obliquus, carbon source, liquid fermentation, polysaccharide synthesis, structural properties, activities

Received: February 4, 2021 Accepted: April 19, 2021

First published online: April 21, 2021

\section{*Corresponding author} Phone: $+27-87287705$ E-mail: mei@mail.hzau.edu.cn

pISSN 1017-7825 elSSN 1738-8872

Copyright(C) 2021 by The Korean Society for Microbiology and Biotechnology

\section{Introduction}

Medicinal fungi have received considerable research attention because of the various bioactivities displayed by their metabolites. These metabolites typically have complex and novel structures, and are an important source of precursors in drug discovery and functional foods. Inonotus obliquus, commonly known as 'chaga,' is a black parasitic fungus (division Basidiomycota, family Hymenochaetaceae) [1] that has been widely used in Russia and Eastern Europe since at least the 16th century as a treatment in folk medicine for cancer, cardiopathy, hepatopathy, gastropathy, and diabetes [2]. Numerous studies have focused on production and pharmacological activities of active metabolites of I. obliquus, which include triterpenoids, polyphenols, fuscoporine, and polysaccharides. In particular, I. obliquus polysaccharides (IOPs) have been found to display notable immunostimulatory [3], anticancer [4], anti-oxidant [5], anti-fatigue [6], and hypoglycaemic [7] activities.

The natural distribution of I. obliquus is mainly between $45^{\circ}$ and $50^{\circ}$ north latitude, which includes Heilongiiang and Jilin provinces of China, Siberia, northern Europe, and Japan. Because of the harsh environments in which I. obliquus grows, wild resources are limited and extremely expensive to obtain. Artificial culture techniques have been developed in response to consumer demand; these include wooden culture, solid-state fermentation, and liquid fermentation. Liquid fermentation, because of its short fermentation period, relatively low cost, and high metabolite production, is a useful method for producing large quantities of I. obliquus metabolites, particularly IOPs. An increasing number of studies during the past decade have focused on optimization of liquid fermentation parameters for enhancing production of I. obliquus mycelial biomass and active metabolite yield. Wei et al. developed a glucose fed-batch integrated dissolved oxygen (DO) control strategy for I. obliquus liquid fermentation that resulted in significant enhancement of biomass and production of polysaccharides, triterpenoids, and inotodiol [1]. In a forced air injection system, a nitrogen:oxygen ratio of 50:50 gave the best 
results in terms of biomass and contents of triterpenes and betulinic acid [8]. Certain stimulatory agents $\left(\mathrm{VB}_{6}\right.$, farnesol, Tween 80) also enhanced mycelial biomass and contents of extracellular polysaccharides, triterpenoids, and betulinic acid in I. obliquus liquid fermentation [9-11]. These and other studies have demonstrated substantial improvement of I. obliquus growth and/or metabolite production by a variety of optimization strategies in terms of fermentation medium and/or processing conditions; however, such studies have generally not addressed the structural characterization or bioactivity of metabolites.

We investigated the effects of fermentation medium components on synthesis, structures, and bioactivities of I. obliquus mycelial polysaccharides generated by liquid fermentation. Glucose (Glc), fructose (Fru), and lactose (Lac) were used as single or combined carbon sources. Crude polysaccharides and neutral polysaccharides were extracted and purified from mycelia based on various carbon sources. These polysaccharide fractions were subjected to structural characterization and comparative analysis of immune-enhancing and anti-tumor effects. In addition, we evaluated the effects of different carbon sources on expression levels of related key enzymes involved in IOP biosynthetic pathway.

\section{Materials and Methods \\ Materials and Reagents}

I. obliquus was obtained from the Microbial Genetic Stock Center of Huazhong Agricultural University (Wuhan, China). DEAE cellulose-52, Sephadex G-100, and T-series dextran molecular weight standards (T-10, T40, T-70, T-500, T-2000) were from Pharmacia (Sweden). D-glucose (Glc), D-mannose (Man), D-arabinose (Ara), D-xylose (Xyl), D-fucose (Fuc), L-rhamnose (Rha), inositol, and erythritol (purity of standards $\geq 99 \%$ ) were from Sigma-Aldrich (USA). DMEM, RPMI-1640, trypsin, penicillin-streptomycin, and diethyl pyrocarbonate (DEPC)-treated water were from Gibco (USA). High-Capacity cDNA Reverse Transcription Kit was from Thermo Fisher Scientific (USA). HiPure Fungal RNA Mini Kit was from Megen (China). HiScript II Q RT SuperMix for qPCR Kit was from Vazyme (China). Cell Counting Kit-8 (CCK-8 Kit) was from Beyotime Institute of Biotechnology (China). Concanavalin A (ConA) and camptothecin (CPT) were from Macklin Biochemical Co. (China) Macrophage RAW264.7, human cervical cancer HeLa, and mouse sarcoma S180 cell lines were from American Type Culture Collection (ATCC; USA). RAW264.7 was cultured with 10\% FBS and 1\% double-strength RPMI-1640, and HeLa and S180 were cultured with $10 \% \mathrm{FBS}$ and $1 \%$ DMEM, for 2 days at $37^{\circ} \mathrm{C}$ in $5 \% \mathrm{CO}_{2}$ atmosphere. Other reagents were from Sinopharm Chemical Reagent Co. (China).

\section{Liquid Fermentation of I. obliquus with Various Carbon Sources}

I. obliquus was inoculated on potato dextrose agar (PDA) slants and cultured for 7 days at $28^{\circ} \mathrm{C}$. Mycelia were harvested and inoculated to seed medium, which consisted of (all following concentrations g/l): Glc (20), malt extract (6), peptone (5), yeast extract (3), $\mathrm{MgSO}_{4} \cdot 7 \mathrm{H}_{2} \mathrm{O}$ (3), $\mathrm{KH}_{2} \mathrm{PO}_{4}(0.4)$, and $\mathrm{K}_{2} \mathrm{HPO}_{4}$ (0.2). Fermentation medium consisted of yeast extract (10), $\mathrm{ZnSO}_{4} \cdot 7 \mathrm{H}_{2} \mathrm{O}(0.1)$, and carbon source (30) at initial pH 5.6. Nine carbon sources were used: Glc, Man, Gal, Xyl, Fru, sucrose (Suc), maltose (Mal), Lac, and starch. For liquid fermentation, mycelium suspension from seed culture (final concentration $10 \%, \mathrm{v} / \mathrm{v}$ ) was inoculated into $100 \mathrm{ml}$ fermentation medium, and cultured for 12 days at $28^{\circ} \mathrm{C}$ with rotation $(160 \mathrm{r} / \mathrm{min})$. Mycelia were harvested, dried to constant weight at $60^{\circ} \mathrm{C}$, ground to powder, and stored at $4^{\circ} \mathrm{C}$. Polysaccharide yield and content were calculated according to the following formulas:

Polysaccharide content $(\mathrm{g} / \mathrm{l})=$ Weight of polysaccharide $(\mathrm{g}) /$ Volume of the fermented broth (L)

Polysaccharide yield $(\%)=[$ Weight of polysaccharide $(\mathrm{g}) /$ Mycelial weight $(\mathrm{g})] \times 100$

\section{Extraction and Purification of I. obliquus Polysaccharides}

Mycelial powder was mixed with hot water (solid/ liquid ratio 1:40, v/v) at $90^{\circ} \mathrm{C}$ for $3 \mathrm{~h}$, deproteinized using $1 / 5$ volume of Sevag solvent (chloroform/ n-butanol 4:1, v/v), and centrifuged $(8,000 \times g)$ for 5 min at room temperature. The top supernatant was collected, precipitated with 4 volumes of ethanol solution, and centrifuged as above. Then, crude I. obliquus polysaccharides (termed CIOPs) were obtained. The CIOP solution was loaded on a DEAE-52 column $(1.5 \times 20 \mathrm{~cm})$ pre-equilibrated with distilled water, and eluted with double-distilled water and $\mathrm{NaCl}$ solution $(0.5 \mathrm{~mol} / \mathrm{l})$ at flow rate $1 \mathrm{ml} / \mathrm{min}$. Eluates were collected, and carbohydrate content was measured by phenol-sulfuric acid method [12]. Material was further purified by gel filtration chromatography using a Sephadex G-100 column $(1.5 \times 60 \mathrm{~cm})$, and then eluted with double-distilled water to yield purified polysaccharide fractions. These polysaccharide fractions were neutral polysaccharides and thus termed as NIOPs. Carbohydrate and protein contents of CIOPs and NIOPs were assayed respectively by phenol-sulfuric acid method and Bradford method [13].

\section{Molecular Weight (MW) and Monosaccharide Composition of NIOPs}

MW of NIOPs was determined using a calibration curve generated by plotting weights of T-series dextran standards against retention time. Monosaccharide compositions of NIOPs were analyzed by gas chromatography (GC) as described by Wang et al. [14] with minor modification. Ten milligrams of each NIOP sample and $2 \mathrm{ml}$ trifluoroacetic acid (TFA) (4 mol/l) were sealed with $\mathrm{N}_{2}$, hydrolyzed at $120^{\circ} \mathrm{C}$ for $3 \mathrm{~h}$, evaporated at $90^{\circ} \mathrm{C}$, and added with methanol to remove excess TFA. Dried hydrolysate $(10 \mathrm{mg})$ was mixed with inositol $(5 \mathrm{mg})$, hydroxylamine hydrochloride $(10 \mathrm{mg})$, and pyridine $(0.5 \mathrm{ml})$, incubated at $90^{\circ} \mathrm{C}$ for $30 \mathrm{~min}$, cooled to room temperature, added with acetic anhydride $(0.5 \mathrm{ml})$, incubated again at $90^{\circ} \mathrm{C}$ for $30 \mathrm{~min}$, and centrifuged 
$(8,000 \times g)$ for $5 \mathrm{~min}$ at room temperature. Supernatant was collected and analyzed by GC (model $6890 \mathrm{~N}$; Agilent Technologies) with phenyl methylsiloxane capillary chromatographic column $(\mathrm{Hp}-5 ; 30 \times 320 \times 0.25 \mu \mathrm{m})$ and flame ionization detector. Column temperature was maintained at $120^{\circ} \mathrm{C}$ for $3 \mathrm{~min}$, and $230^{\circ} \mathrm{C}$ for $4 \mathrm{~min}$. Monosaccharide standards were analyzed by GC procedure as above.

\section{Fourier Transform Infrared Spectroscopy (FTIR)}

Dried NIOP samples $(2 \mathrm{mg})$ were mixed with $\mathrm{KBr}(200 \mathrm{mg})$ and ground to thin pellets in an agate mortar under infrared lamp. The thin section was analyzed using a Nicolet Nexus FTIR 470 spectrophotometer (Thermo Scientific Nicolet; USA) over wavelength range 400 to $4,000 \mathrm{~cm}^{-1}$ [15].

\section{Periodate Oxidation and Smith Degradation}

Each NIOP sample was subjected to periodate oxidation and Smith degradation by the method of Zhang et al. [16]. A twenty-milligram NIOP sample was dissolved with $20 \mathrm{ml} \mathrm{NaIO}_{4}(15 \mathrm{mM})$ at $4^{\circ} \mathrm{C}$ in the dark. Reaction solution was added with ethylene glycol $(0.4 \mathrm{ml})$, and absorbance measured by spectrophotometry at wavelength $223 \mathrm{~nm}$ at 24-h intervals. $\mathrm{HIO}_{4}$ consumption and formic acid production were assayed by titration with $\mathrm{NaOH}$ $(10 \mathrm{mM})$. Periodate product was added with ethylene glycol $(2 \mathrm{ml})$, stirred for $30 \mathrm{~min}$, dialyzed with flowing water for $24 \mathrm{~h}$ and then distilled water for $24 \mathrm{~h}$, and concentrated to a volume of $10 \mathrm{ml}$. The resulting concentrated solution was mixed with $50 \mathrm{mg} \mathrm{NaBH}_{4}$, stirred in the dark at room temperature for $24 \mathrm{~h}$, $\mathrm{pH}$-adjusted to 5.5 with $0.1 \mathrm{M}$ acetic acid, dialyzed, and evaporated. The product was subjected to GC analysis as described above.

\section{In Vitro Cell Viability Assay}

Immune-enhancing activity of IOPs was evaluated using RAW264.7 cells. Log-phase cells were added with RPMI- 1640 to concentration $5 \times 10^{5} / \mathrm{ml}$. Cell suspensions $(100 \mu \mathrm{l})$ were mixed with $10 \mu \mathrm{l}$ CIOPs at concentration $10-500 \mu \mathrm{g} / \mathrm{ml}$ or with NIOPs at concentration $1-100 \mu \mathrm{g} / \mathrm{ml}$, and incubated on 96 -well plates in $5 \% \mathrm{CO}_{2}$ atmosphere at $37^{\circ} \mathrm{C}$. After $48 \mathrm{~h}$, the mixture was added with $10 \mu \mathrm{CCK}-8$ solution, incubated for $3 \mathrm{~h}$, and $\mathrm{OD}_{450}$ was measured. Positive control was treated with ConA at concentration $1 \mu \mathrm{g} / \mathrm{ml}$, and normal control was not treated with any drug. Treatments were performed in triplicate. Cell viability was calculated as:

Cell viability $(\%)=\left[\mathrm{OD}_{450}(\right.$ sample $)-\mathrm{OD}_{450}($ control $\left.)\right] \times 100$

Anti-tumor activity was evaluated using HeLa and S180 cells. IOP samples (final concentration 500 and $1,000 \mu \mathrm{g} / \mathrm{ml})$ and $\mathrm{HeLa} / \mathrm{S} 180$ cells $\left(5 \times 10^{4} / \mathrm{ml}\right)$ were cultured on a 96 -well plate for $24 \mathrm{~h}$ at $37^{\circ} \mathrm{C}$. CPT at concentration $600 \mu \mathrm{g} / \mathrm{ml}$ was used for positive control. Cell viabilities were determined by CCK- 8 kit and calculated by the above formula.

\section{Quantitative Real-Time PCR (qRT-PCR)}

Transcription levels of phosphoglucose isomerase (PGI), UDP-Glc 4-epimerase (UGE) and $\beta$-actin (reference) genes were analyzed by qRT-PCR. I. obliquus mycelial powder $(50 \mathrm{mg})$ was prepared for extraction of RNA using a HiPure Fungal RNA Mini Kit. Total generated RNA was dissolved in diethylpyrocarbonate (DEPC) water and transcribed to cDNA as per the HiScript IIQ RT SuperMix protocol for the qPCR kit. The resulting cDNA was used as template. Primer sequences were $\left(5^{\prime} \rightarrow 3^{\prime}\right)$ : PGI: F: ACCACGCAGGAGACGATCAC, R: TGTTGTCAGCCGAGA TACCA; UGE: F: TCGGGAACGACTATCCGAC, R: AGCCTTAAACCGTCCTGTAC; $\beta$-actin: F: CCACGA GACAACATACAACT, R: TACCACCAGACAGCACAAC. The qRT-PCR program was: $95^{\circ} \mathrm{C}$ for 3 min (stage 1); 40 cycles of $95^{\circ} \mathrm{C}$ for $10 \mathrm{sec}, 60^{\circ} \mathrm{C}$ for $20 \mathrm{sec}$ (stage 2); and $95^{\circ} \mathrm{C}$ for $15 \mathrm{sec}, 60^{\circ} \mathrm{C}$ for $60 \mathrm{sec}$, and $95^{\circ} \mathrm{C}$ for $15 \mathrm{sec}$ (stage 3). Reactions were performed in triplicate. Relative expression levels of PGI and UGE were normalized and determined by $2^{-\Delta \Delta C t}$ method [17].

\section{Statistical Analysis}

All experiments were performed in triplicate. Data were expressed as mean $\pm \mathrm{SD}$, and differences between means were analyzed using software program SPSS for Windows, V. 20 (SPSS, Inc.; USA). Datasets involving more than two groups were assessed by one-way ANOVA followed by Duncan's multiple range test, and differences indicated by differing superscript letters were considered statistically significant $(p<0.05)$. Comparisons between means of two groups were analyzed using Student's $t$-test, and differences with $p<0.05$ or $p<0.01$ were considered significant or highly significant, respectively [18].

\section{Results}

Effects of Carbon Sources on Mycelial Biomass and Polysaccharide Production of I. obliquus

I. obliquus was cultured on optimized medium, using Glc, Fru, Man, Gal, Xyl, Suc, Mal, Lac or starch as carbon source, and mycelial biomass and polysaccharide content were measured on day 12. Highest biomass values were observed when sole carbon source was Glc, Fru, Man, or Xyl (Fig. 1A). Both biomass and polysaccharide content were high for the Glc group, indicating that Glc is a good carbon source for mycelial growth and polysaccharide production. Although Lac, starch, Mal, and Gal groups had lower biomass values, their polysaccharide yields were higher than those of Glc, Fru, Man, and Xyl groups. We therefore performed experiments with a "mixotrophic" strategy using Glc and a supplementary carbon source in molar ratio 2:1. Mycelial biomass, polysaccharide yield, and polysaccharide content were all significantly higher for the Glc+Lac group (Glc and Lac [2:1] used as combined carbon source) than for other mixotrophic groups (Fig. 1B). 
A

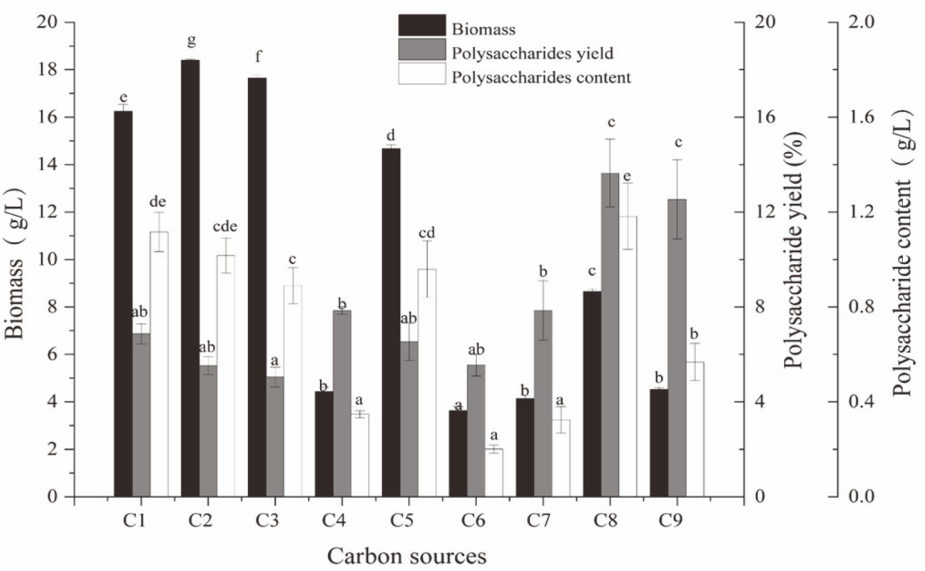

B



Fig. 1. Effects of carbon source on biomass and polysaccharide production of Inonotus obliquus. (A) Single carbon sources. (B) Complex carbon sources. C1-C9: Glc, Fru, Man, Gal, Xyl, Suc, Mal, Lac, and starch; C10-C15: Glc, Glc + Gal, Glc + Suc, Glc + Mal, Glc + Lac, Glc + starch. Differing letters above bars indicate significant $(p<0.05)$ differences according to Duncan's multiple range test.

Isolation and Purification of Polysaccharides from I. obliquus Mycelia

I. obliquus was grown for 12 days by liquid fermentation using Glc, Fru, Lac, or Glc+Lac as carbon source, mycelia were harvested, and polysaccharides were isolated by hot water extraction under optimal conditions. Four crude I. obliquus polysaccharides (CIOPs) were generated, and termed CIOPG (Glc as carbon source), CIOPF (Fru as carbon source), CIOPL ( $\mathrm{Lac}$ as carbon source), and CIOPGL (Glc+Lac as carbon source). Each of the above four CIOPs was separated by DEAE- 52 column chromatography into two fractions, i.e., CIOPG-1 and -2 (Fig. 2A), CIOPF-1 and -2 (Fig. 2B), CIOPL-1 and -2 (Fig. 2C), and CIOPGL-1 and -2 (Fig. 2D). Each of the above

Table 1. Properties of crude polysaccharides and neutral polysaccharides from I. obliquus mycelia.

\begin{tabular}{|c|c|c|c|c|c|}
\hline Polysaccharides & $\begin{array}{l}\text { Carbohydrate } \\
(\%)\end{array}$ & $\begin{array}{l}\text { Protein } \\
\quad(\%)\end{array}$ & $\begin{array}{l}\text { Molecular } \\
\text { weight } \\
(\mathrm{kDa})\end{array}$ & $\begin{array}{c}\text { Monosaccharide } \\
\text { composition } \\
\text { (molar ratio) }\end{array}$ & Glycosidic linkages \\
\hline CIOPG & $33.48 \pm 0.93$ & $3.48 \pm 0.74$ & - & - & - \\
\hline CIOPF & $18.88 \pm 0.57$ & $1.59 \pm 0.57$ & - & - & - \\
\hline CIOPL & $20.77 \pm 0.85$ & $0.96 \pm 1.13$ & - & - & - \\
\hline CIOPGL & $22.83 \pm 1.44$ & $1.01 \pm 1.52$ & - & - & - \\
\hline NIOPG & $98.39 \pm 1.83$ & $0.23 \pm 1.12$ & 780.90 & $\begin{array}{l}\text { Glc, Man, Gal } \\
(92.17: 4.49: 3.35)\end{array}$ & $\begin{array}{l}1 \rightarrow 2,1 \rightarrow 3,1 \rightarrow 4,1 \rightarrow 6 \\
(3.52 \% / 32.39 \% / 47.08 \% / 17.01 \%)\end{array}$ \\
\hline NIOPF & $96.21 \pm 1.60$ & $0.83 \pm 0.07$ & 1105.00 & $\begin{array}{l}\text { Glc, Man, Gal } \\
(68.73: 18.33: 12.94)\end{array}$ & $\begin{array}{l}1 \rightarrow 2,1 \rightarrow 3,1 \rightarrow 4,1 \rightarrow 6 \\
(3.05 \% / 38.45 \% / 32.17 \% / 26.33 \%)\end{array}$ \\
\hline NIOPL & $90.60 \pm 2.28$ & $0.13 \pm 0.29$ & 25.32 & $\begin{array}{l}\text { Glc, Man, Gal } \\
(8.98: 52.36: 38.12)\end{array}$ & $\begin{array}{l}1 \rightarrow 2,1 \rightarrow 3,1 \rightarrow 4,1 \rightarrow 6 \\
(49.64 \% / 27.04 \% / 4.69 \% / 18.63 \%)\end{array}$ \\
\hline NIOPGL & $90.96 \pm 1.76$ & $0.35 \pm 0.22$ & 10.28 & $\begin{array}{l}\text { Glc, Man, Gal } \\
(26.43: 42.18: 31.38)\end{array}$ & $\begin{array}{l}1 \rightarrow 2,1 \rightarrow 3,1 \rightarrow 4,1 \rightarrow 6 \\
(34.20 \% / 29.47 \% / 15.27 \% / 21.06 \%)\end{array}$ \\
\hline
\end{tabular}


A

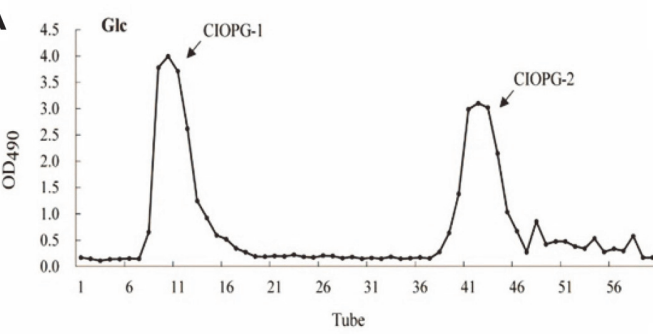

B

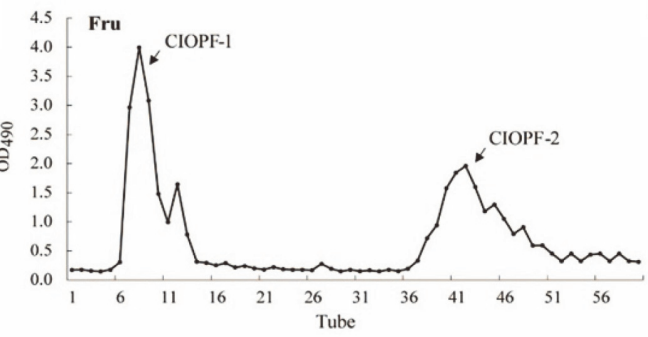

C

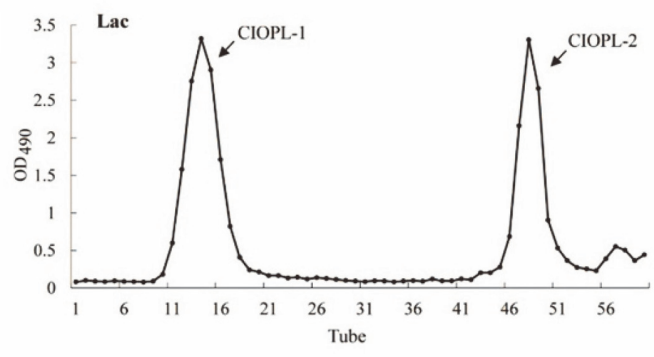

D

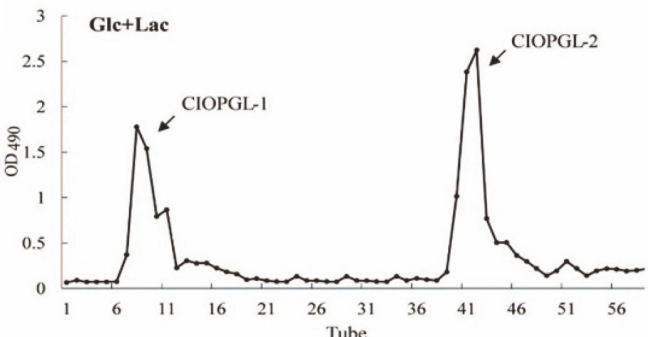

E

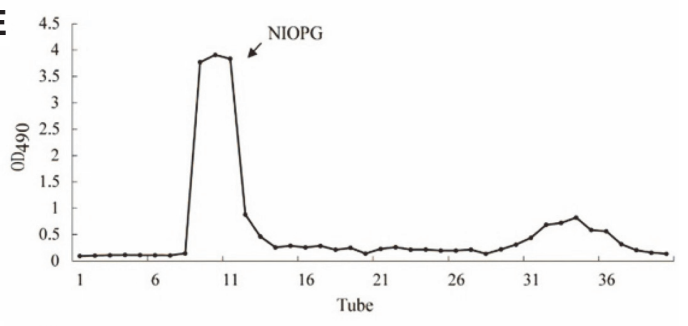

$\mathbf{F}$

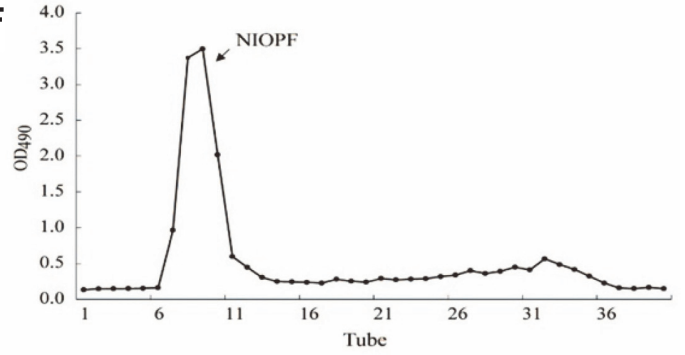

G
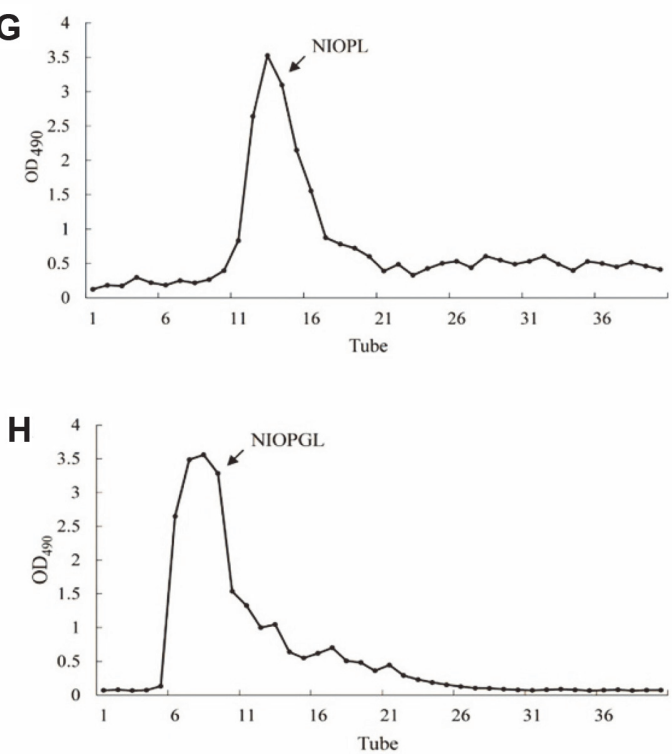

Fig. 2. Isolation and purification of crude polysaccharides (A-D) and neutral polysaccharides (E-F) from I. obliquus mycelia. Carbon sources used were Glc (A,E), Fru (B,F), Lac (C,G), and Glc+Lac (D,H).

-1 fractions was eluted with water and classified as neutral sugar, and each -2 fraction was eluted with $0.5 \mathrm{M} \mathrm{NaCl}$ solution and classified as acid sugar. The four CIOPs differed in regard to contents and ratios of neutral and acid sugars (Figs. 2A-2D).

Because of the abundance of neutral polysaccharides in the four CIOPs, the - 1 fractions were further purified by Sephadex G-100 column chromatography. Four purified neutral polysaccharides (NIOPs) were thus generated, and termed NIOPG, NIOPF, NIOPL, and NIOPGL (Figs. 2E-2H). Carbohydrate contents differed notably among the four CIOPs and four NIOPs (Table 1).

\section{Effects of Carbon Source on Structural Properties of I. obliquus Mycelial Polysaccharides}

Carbohydrate content of each of the four NIOPs was $>90 \%$, much higher than values for the corresponding CIOPs, which ranged from $18.88 \%$ to $33.48 \%$ (Table 1). The NIOPs were therefore used for analysis of structural properties. They had typical carbohydrate spectra upon FTIR analysis (Fig. 3). These spectra had similar peaks at 3,327.78-33,615.66 cm $\mathrm{cm}^{-1}$ (O-H stretching), 2,917.08-2,918.74 $\mathrm{cm}^{-1}$ (C-H stretching), $1,644.03-1,646.91 \mathrm{~cm}^{-1}(\mathrm{C}=\mathrm{O}$ stretching), $1,351.22-1,353.22 \mathrm{~cm}^{-1}$ (asymmetrical C-H bending of $\mathrm{CH}_{2}$ group), and 1,014.73-1,147.73 $\mathrm{cm}^{-1}$ (C-OC) [19]. Absorption peaks at $575.89-593.95 \mathrm{~cm}^{-1}$ were attributed to $\alpha$-glycosidic bond [14]. Three peaks around $1000-1200 \mathrm{~cm}^{-1}$ in NIOPG and NIOPF indicated the presence of pyranose (Figs. 3A and 3B). In contrast, NIOPL and NIOPGL had two peaks in that range, suggesting the presence of furanose (Figs. 3C and 3D).

MW values of the four NIOPs were determined as 780.90 (NIOPG), 1105.00 (NIOPF), 25.32 (NIOPL), and $10.28 \mathrm{kDa}$ (NIOPGL), based on retention time relative to a series of standard glucans (Table 1). Monosaccharide 
A

B

C

NIOPG
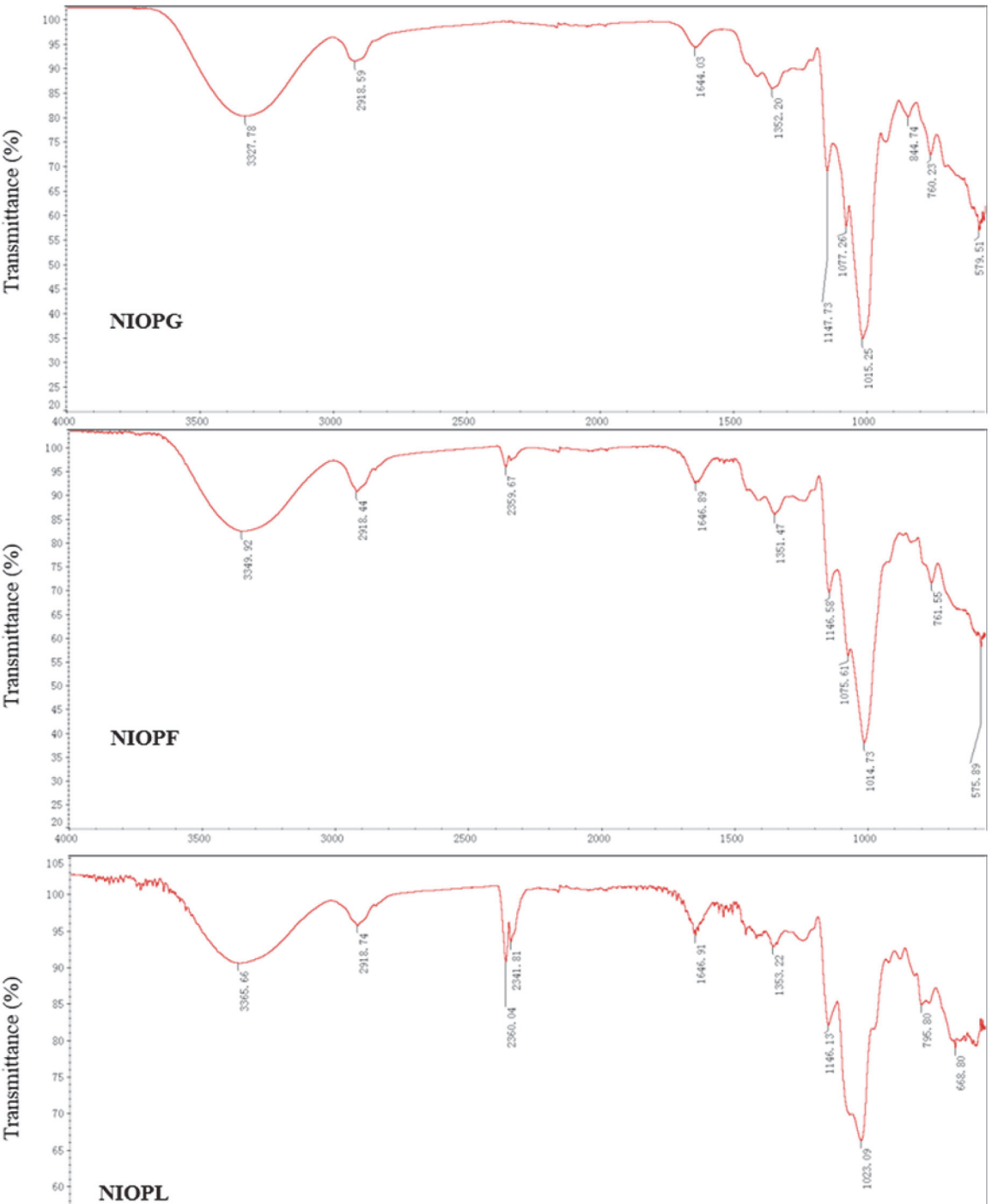

D

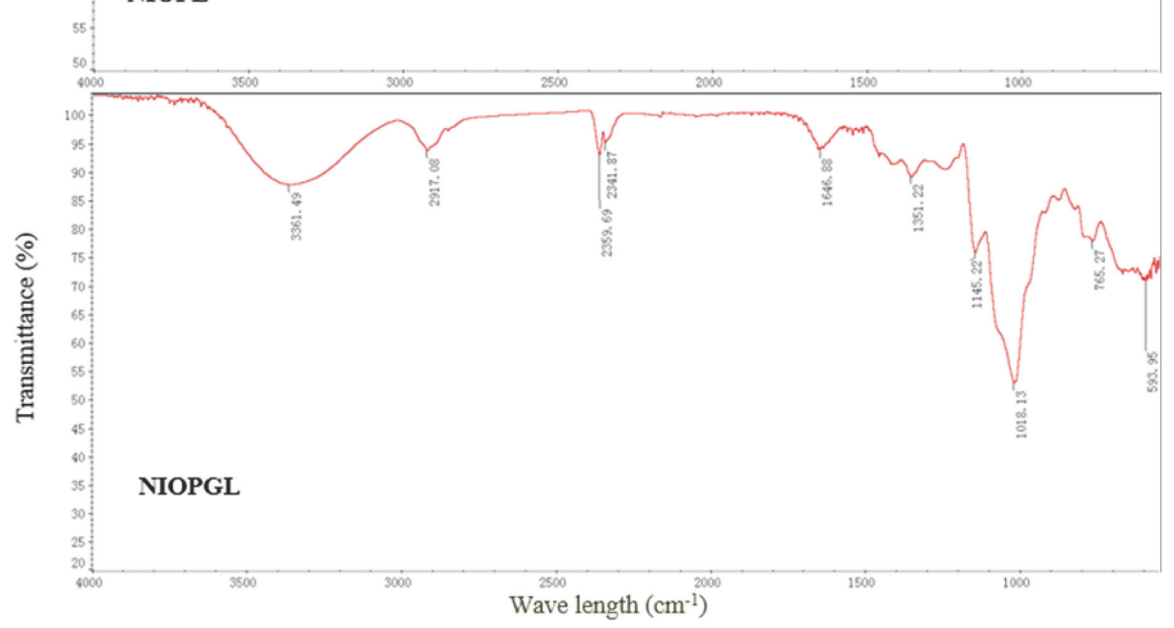

Fig. 3. FTIR spectra of NIOPG (A), NIOPF (B), NIOPL (C), and NIOPGL (D).

compositions were determined by GC-MS, and profiles are shown in Fig. 4. Compositions of the four NIOPs were found to include Glc, Man, and Gal, Based on comparison with retention times of seven saccharide standards (inositol used as internal reference), we concluded that NIOPs were composed of Glc, Man, and Gal in respective molar ratios 92.17: 4.49: 3.35 (NIOPG), 68.73:18.33: 12.94 (NIOPF), 8.98: 52.36: 38.12 (NIOPL), and 26.43: 42.18: 
A

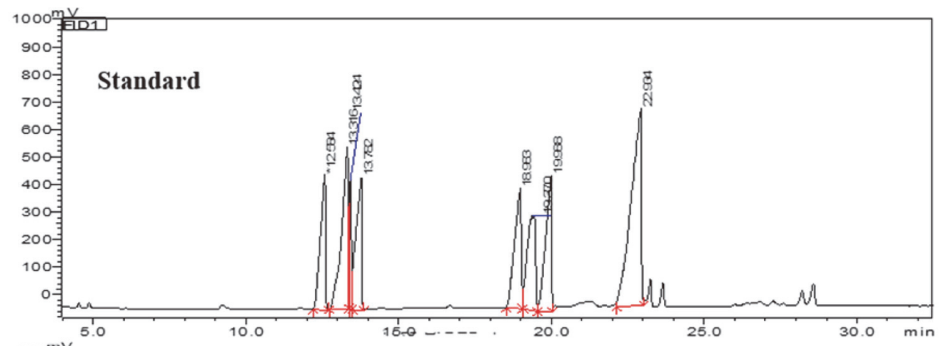

B

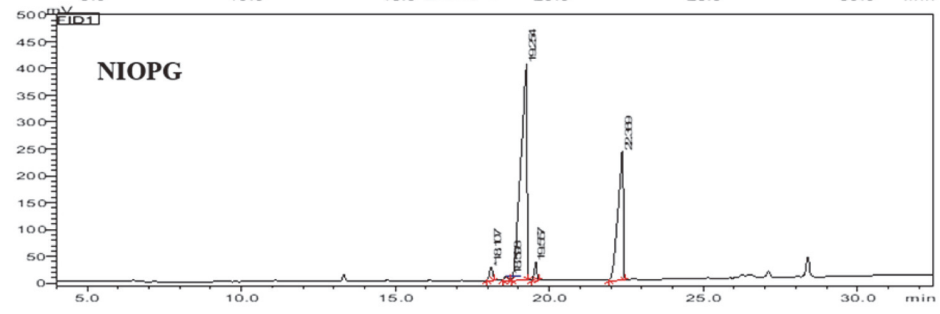

C

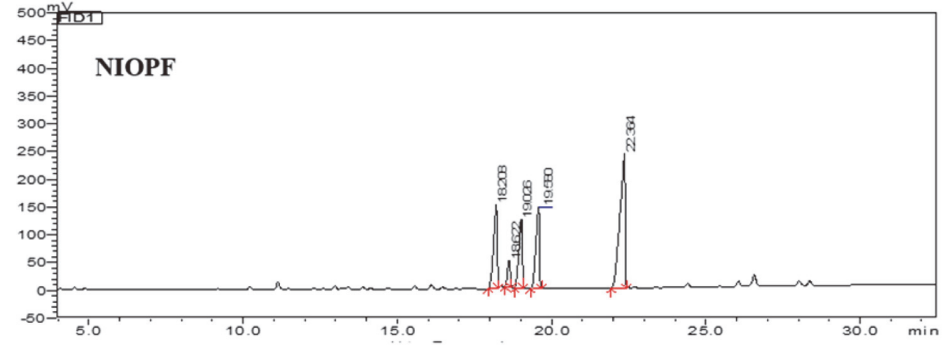

D

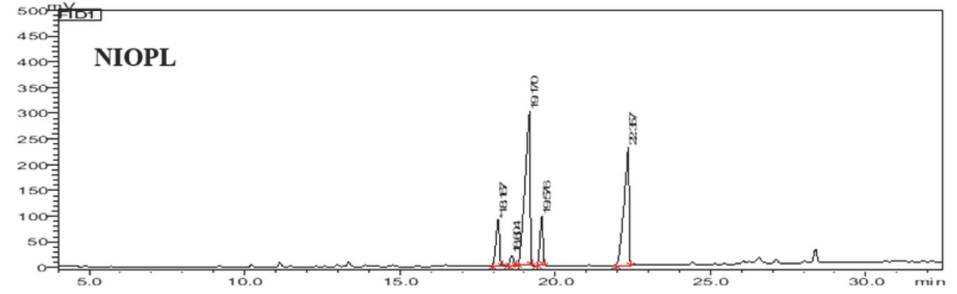

E

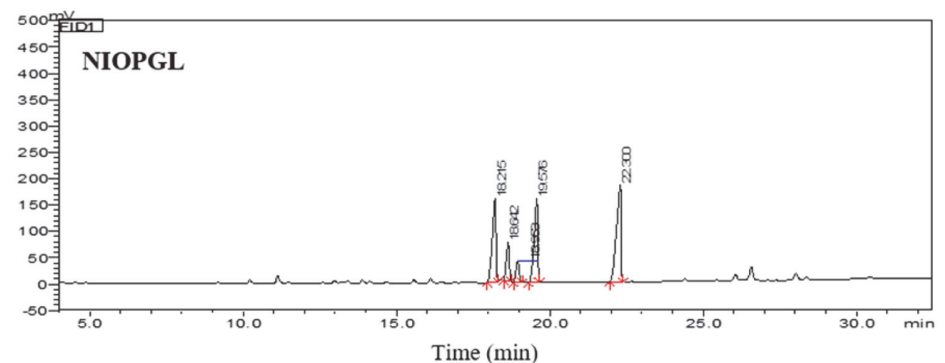

Fig. 4. GC-MS analysis of standard monosaccharide mixture (A) and hydrolysis products of NIOPG (B), NIOPF (C), NIOPL (D), and NIOPGL (E). A: standard monosaccharides including Rha, Ara, Fuc, Xyl, Man, Glc, Gal; inositol used as internal reference.

\subsection{8 (NIOPGL) (Table 1).}

NIOPs were subjected to periodate oxidation and Smith degradation, and glycosidic linkages were analyzed by GC-MS. Periodate consumption values for NIOPG, NIOPF, NIOPL, and NIOPGL were respectively 0.8462, $0.8787,0.9159$, and $0.9159 \mathrm{~mol} / \mathrm{mol} \mathrm{Glc}$, indicating presence of $1 \rightarrow 2,1 \rightarrow 4$, and/or $1 \rightarrow 6$ linkages. Formic acid yields were $>0$ for each of the NIOPs, indicating presence of $1 \rightarrow 6$ linkage (Table 2). Smith degradation products were determined by GC-MS to be glycerol: Glc: erythritol in molar ratios 0.5107: 0.1142: 1 (NIOPG), 1.0078: 0.6420: 1(NIOPF), 25.1345: 16.9553: 1 (NIOPL), and 5.8569: 4.8408: 1 (NIOPGL). Proportions of $1 \rightarrow 2,1 \rightarrow 3,1 \rightarrow 4$, and $1 \rightarrow 6$ linkages were calculated, based on these values, as 3.52/32.39/47.08/ 17.01\% (NIOPG), 3.05/ 38.45/ 32.17/ $26.33 \%$ (NIOPF), 49.64/ 27.04/ 4.69/ 18.63\% (NIOPL), and 34.20/ 29.47/ 15.27/ 21.06\% (NIOPGL) (Table 1). In conclusion, structural properties differed greatly among the four NIOPs, indicating a strong effect of carbon source on structures of I. obliquus mycelial polysaccharides. 
Table 2. Results of periodate oxidation/Smith degradation of Inonotus obliquus neutral polysaccharides.

\begin{tabular}{lccl}
\hline Linkage & $\begin{array}{c}\text { Periodate consumption } \\
(\mathrm{mol} / \mathrm{mol} \text { Glc })\end{array}$ & $\begin{array}{c}\text { Formic acid production } \\
(\mathrm{mol} / \mathrm{mol} \text { Glc })\end{array}$ & \multicolumn{1}{c}{ Smith degradation products } \\
\hline $1 \rightarrow 2$ & 1 & $\mathrm{nd}^{\mathrm{a}}$ & Glycerol \\
$1 \rightarrow 3$ & $\mathrm{nd}$ & $\mathrm{nd}$ & Glucose/mannose \\
$1 \rightarrow 4$ & 1 & $\mathrm{nd}$ & Erythritol \\
$1 \rightarrow 6$ & 2 & 1 & Glycerol \\
NIOPG & 0.8462 & 0.1701 & Glycerol: Glucose: Erythritol=0.5107:0.1142:1 \\
NIOPF & 0.8787 & 0.2632 & Glycerol: Glucose: Erythritol=1.0078:0.6420:1 \\
NIOPL & 0.9159 & 0.1863 & Glycerol: Glucose: Erythritol $=25.1345: 16.9553: 1$ \\
NIOPGL & 0.9159 & 0.2106 & Glycerol: Glucose: Erythritol $=5.8569: 4.8408: 1$ \\
\hline
\end{tabular}

${ }^{a}$ not detected.

Effects of Carbon Source on Activities of I. obliquus Mycelial Polysaccharides

The effects of CIOPs and NIOPs on proliferation of macrophage cells (RAW264.7) were evaluated in vitro, using ConA $(1 \mu \mathrm{g} / \mathrm{ml})$ as positive control. The four CIOPs, at concentration 10-500 $\mu \mathrm{g} / \mathrm{ml}$, had a significant $(p<0.05$ or $<0.01)$, dose-dependent enhancing effect on proliferation in comparison with control (Fig. 5A). The same was true for the four NIOPs (Fig. 5B). Proliferation-enhancing effect in concentration range $1-100 \mu \mathrm{g} / \mathrm{ml}$ was stronger for NIOPs than for CIOPs, indicating that such activity was due to polysaccharides in CIOPs, not to other components. In this low concentration range, RAW264.7 cell viability was enhanced more strongly by NIOPL or NIOPGL than by NIOPG, NIOPF, or ConA (positive control) (Fig. 5B). These findings indicate that I. obliquus mycelial polysaccharides exert immune-enhancing activity, and that this activity is affected by carbon source used in fermentation.

Anti-tumor activities of CIOPs and NIOPs were comparatively assessed based on effect on proliferation of HeLa and S180 cells in vitro, with CPT $(600 \mu \mathrm{g} / \mathrm{ml})$ as positive control. CIOPF and CIOPGL had significant $(p<0.01)$, dose-dependent proliferation-inhibitory effect on HeLa at all tested concentrations (Fig. 6A). At the highest concentration, proliferation-inhibitory effects of CIOPG, CIOPF, and CIOPGL on HeLa were stronger than that of CPT, and effects of CIOPs were stronger than those of NIOPs, suggesting that other components in CIOPs displayed anti-tumor activity. Proliferation-inhibitory effects of NIOPG and NIOPGL on HeLa were stronger than those of NIOPF and NIOPL, consistent with results for S180 cells (Fig. 6B). These findings indicate that I. obliquus mycelial polysaccharides display substantial anti-tumor activity, and that such activity is affected by carbon source.

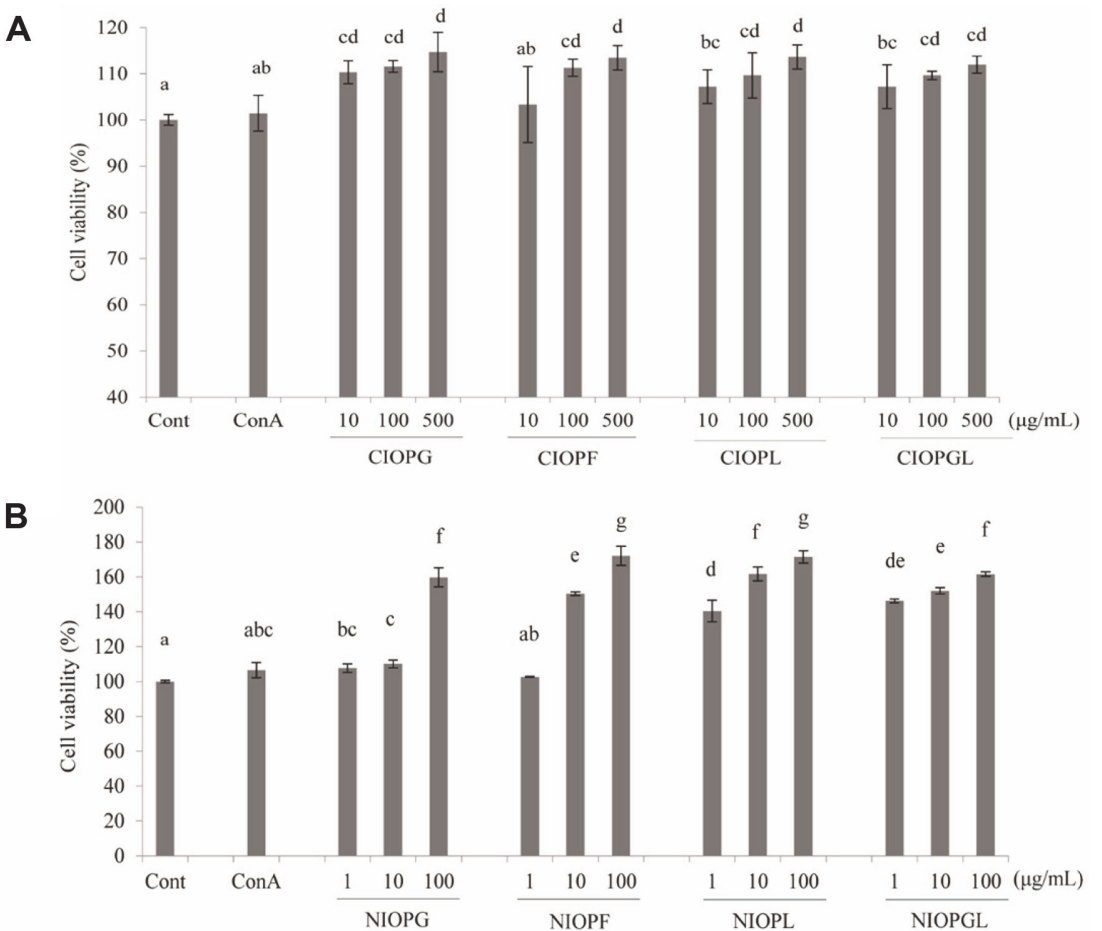

Fig. 5. Effects of crude polysaccharides (A) and neutral polysaccharides (B) on proliferation of RAW264.7 cells in vitro. Cont: normal control group. ConA: positive control group, treated with $1 \mu \mathrm{g} / \mathrm{ml}$. Differing letters above bars indicate significant $(p<0.05)$ differences according to Duncan's multiple range test. 
A

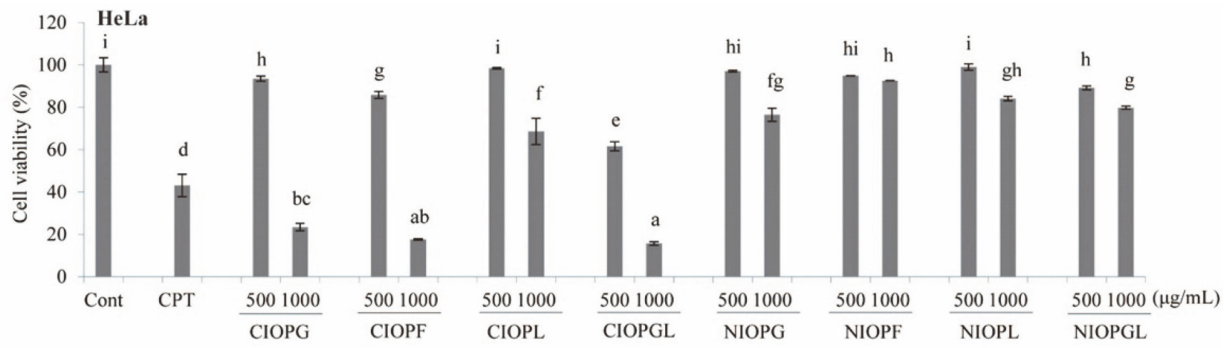

B

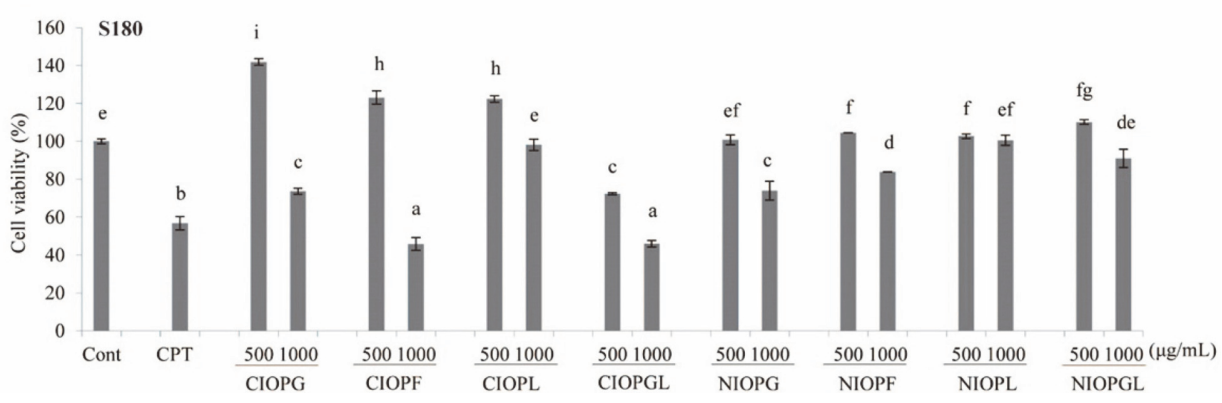

Fig. 6. Effects of crude polysaccharides and neutral polysaccharides on proliferation of HeLa (A) and S180 (B) cells in vitro. Notations as in Fig. 5.

A

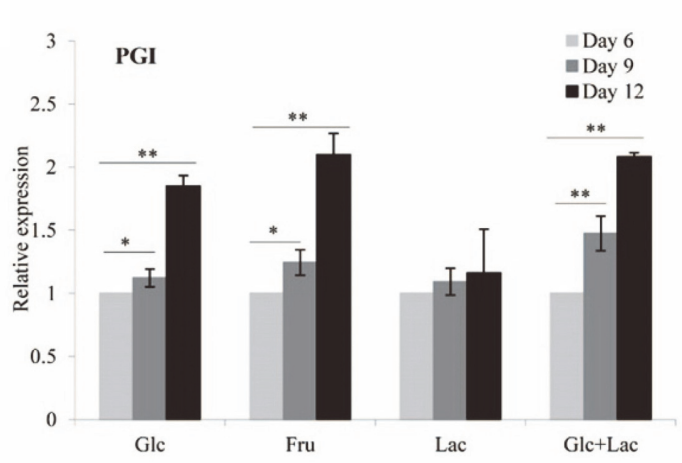

B

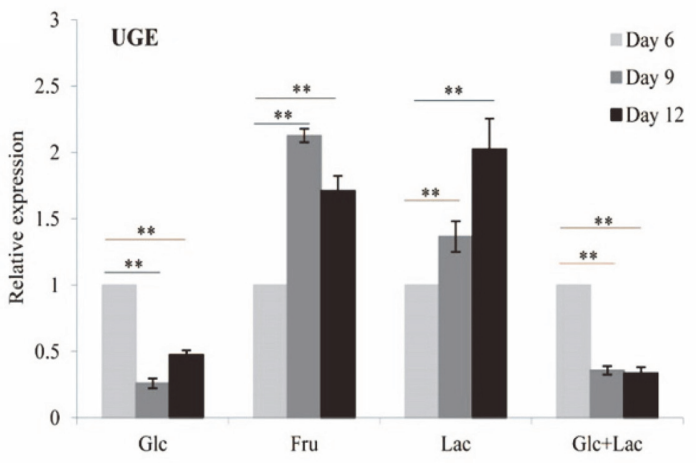

C

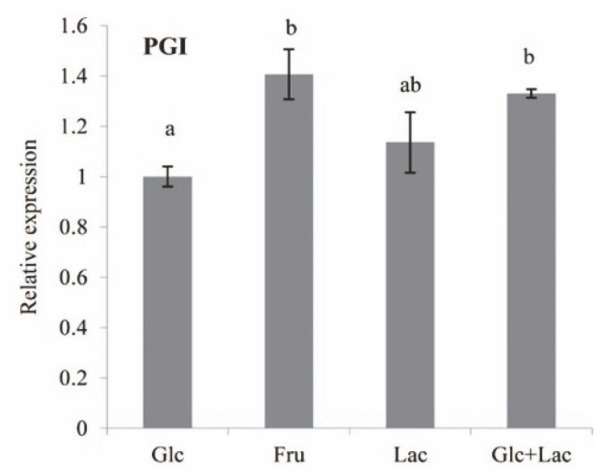

D

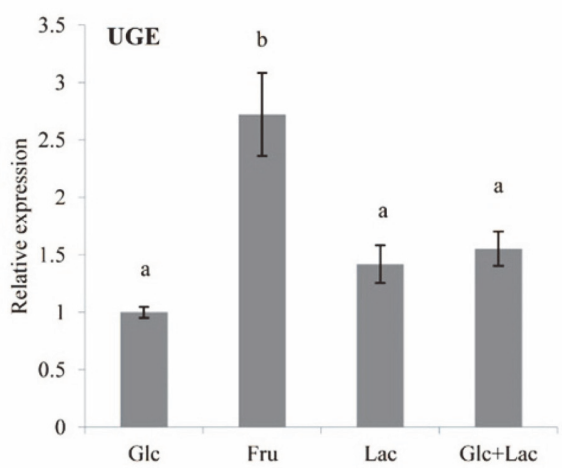

Fig. 7. Effects of carbon source on transcription levels of genes encoding enzymes PGI (A) and UGE (B) involved in I. obliquus polysaccharide biosynthetic pathway. ${ }^{*} p<0.05,{ }^{* *} p<0.01$ vs. Day 6 . Differing letters above bars indicate significant $(p<0.05)$ differences according to Duncan's multiple range test.

Effects of Carbon Source on Expression of Key Genes Involved in Mycelial Polysaccharide Biosynthetic Pathway

To evaluate the relationship between polysaccharide synthesis and carbon source used in fermentation, we analyzed expression levels of genes encoding PGI and UGE, two key enzymes involved in polysaccharide 
biosynthetic pathway. During a 12-d fermentation period, expression level of pgi in the four carbon source groups increased with time (Fig. 7A), consistent with the trend of increasing CIOP content. In the Glc, Fru, and Glc+Lac groups, this increase was significant. Therefore, PGI is evidently a key enzyme in regulation of polysaccharide synthesis, particularly for carbon sources Glc, Fru, or Glc+Lac. The order of pgi expression level at 12 days in the four groups was Fru $>$ Glc $+\mathrm{Lac}>\mathrm{Lac}>\mathrm{Glc}$ (Fig. 7B), indicating a notable effect of carbon source on pgi expression.

In contrast, uge expression level did not vary notably regardless of whether the carbon source was Glc, Fru, or Glc+Lac (Fig. 7C). uge expression level showed significant time-dependent increase only for the Lac group, indicating that CIOPL synthesis is regulated mainly by UGE. uge expression level at the end of the 12-day period was significantly higher for Fru group than for the other three groups (Fig. 7D), indicating a notable effect of carbon source on uge expression.

\section{Discussion}

Microbial polysaccharides (MPs) are secondary metabolites that display a variety of useful functions and have been widely applied in the food and pharmaceutical industries. Relationships among carbon sources, microbial biomass, and polysaccharide yield have long been a topic of great research interest for development and improvement of MP resources and related products [20-23]. In the present study, I. obliquus mycelial biomass and polysaccharide yield were investigated using different carbon sources. The results showed that these two indices were significantly higher for the Glc+Lac group (Glc and Lac used in combination as carbon source) than for other groups. Actually, nutritional carbon source requirements clearly differ for different medicinal fungi. For instance, in the research of mushroom Phellinus linteus, maximal mycelial growth and production of extracellular polysaccharides (EPs) were achieved when sorbitol was used as carbon source [24]. In the truffle Tuber sinense, use of Lac as carbon source promoted EP production, but not mycelial growth [20].

Artificial culture or fermentation techniques are often used for development of bioactive agents based on microbial compounds. In this context, structure and function of polysaccharides are more important than biomass and product yield. Streptococcus pneumoniae, when grown with Fru as carbon source, was unable to synthesize capsular polysaccharide, an important virulence factor [25]. Bacillus subtilis, a widely distributed and researched bacterium, when grown with burdock oligofructose as carbon source, produced a novel polysaccharide that displayed anti-cancer activity [26]. In this study, CIOPs and NIOPs generated by liquid fermentation using differing carbon sources, varied greatly in their immune-enhancing and anti-tumor activities. NIOPL and NIOPG displayed the strongest immune-enhancing and anti-tumor activities, respectively, suggesting potential application in drug or health food development.

Functions of MPs vary depending on structural differences (e.g., monosaccharide composition, glycosidic linkages) which are affected by culture medium components, particularly carbon source. Peng et al. reported that mole percentages of four Ganoderma lucidum EPs (monosaccharides, Glc, Gal, Man) varied depending on mixed carbon source (Glc+Gal or Glc+Man, in molar ratio 1:1 or 1:2) [27]. Monosaccharide composition of Nostoc flagelliforme EPs similarly varied depending on carbon source condition $\left(\mathrm{NaHCO}_{3}\right.$ in concentrations ranging from $0-2.94 \mathrm{~g} / \mathrm{l}$ ) [28]. Kim, Lee and Yun observed that the composition of zooglan, an EP produced by the bacterium Zoogloea ramigera, varied depending on carbon source, with resulting changes in heavy metal adsorption characteristics [29]. In the present study, use of different carbon sources (Glc, Fru, Lac, Glc+Lac) greatly altered MW, monosaccharide composition, and glycosidic linkages of four NIOPs, resulting in varied immune-enhancing and anti-tumor activities.

Variations in structures and functions of MPs are generally associated with their biosynthetic pathways, in which relevant enzymes are up- or downregulated depending on carbon source. Enzymes involved in MP synthetic pathways clearly play key roles in production and structures of MPs [30]. In the case of I. obliquus, the

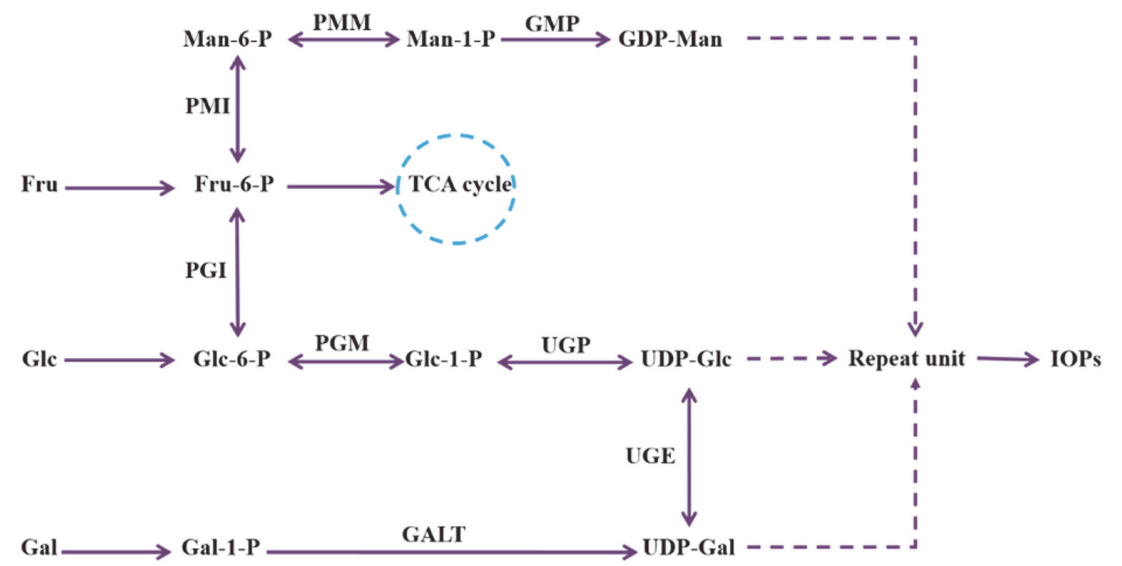

Fig. 8. Proposed biosynthetic pathway for I. obliquus polysaccharides. PMM: phosphomannose mutase; GMP: GDP-Man pyrophosphorylase; PMI: phosphomannose isomerase; PGI: phosphoglucose isomerase; PGM: phosphoglucose mutase; UGP: UDP-Glc pyrophosphorylase; UGE: UDP-Gal-4-epimerase; GALT: galactose-1-phosphate uridylyltransferase. 
IOP biosynthetic pathway, including a series of related enzymes (phosphomannose mutase [PMM], GDP-Man pyrophosphorylase [GMP], phosphomannose isomerase [PMI], PGI, UGE, and UDP-Glc pyrophosphorylase [UGP]), was deduced by monosaccharide compositions of IOPs and carbon sources used in this study (Fig. 8).

In this pathway, PGI represents an important control point and determines the conversion direction of Glc-6phosphate (Glc-6-P), the precursor of sugar nucleotide UDP-Glc. Zhu et al. observed a strong correlation between PGI activity and production of intracellular polysaccharides in the medicinal fungus Cordyceps militaris [31]. PGI level may fluctuate under the fermentation condition using different sugars as carbon sources. In the research of Ganoderma lucidum strain 5.26, PGI level was lower when Suc (in comparison with Glc) was used as sole carbon source [32]. In this study, pgi gene expression under all four carbon source conditions was upregulated as fermentation time increased, consistent with the trend of IOP content. PGI is evidently a key enzyme in IOP synthetic pathway. pgi expression level was higher in Fru group than in other groups, and was correlated with high molar ratio of Glc in NIOPF monosaccharide composition. Under high-Fru condition, PGI catalyzes conversion of Fru-6-P to Glc-6-P. Similarly, NIOPL and NIOPGL contain high proportions of Man and its derivatives, as a result of high expression level of $p g i$, which catalyzes interconversion between Glc-6-P and Fru-6-P. Fru-6-P can be further converted to Man-6-P, Man-1-P, and consequent sugar nucleotide GDP-Man, by catalysis with related enzymes PMI, PMM, and GMP.

UGE level determines the direction of interconversion between UDP-Glc and UDP-Gal, which are precursors of polysaccharide repeat unit. Han et al. observed that UGE activity involved in N. flagelliforme EP synthesis was significantly inhibited when $\mathrm{NaHCO}_{3}$ at concentration $2.94 \mathrm{~g} / \mathrm{l}$ was used as carbon source [28]. In the present study, UGE activity likewise varied depending on carbon source. uge expression level in Lac group was upregulated as fermentation time increased, indicating an important role of UGE in CIOPL synthesis. uge expression level in Glc, Fru, and Glc+Lac groups did not have similar effects, indicating that synthesis of corresponding IOPs may be associated with regulation of other key genes. Peng et al. reported correlation of higher Gal molar ratio in monosaccharide composition of G. lucidum EPs with higher PGM activity [33]. In $N$. flagelliforme EPs, higher proportion of Gal was associated with higher UDP-Glc dehydrogenase (UDPD) activity [28]. Relationships between carbon source and regulation of IOP synthesis will be clarified by future studies of other relevant enzymes in IOP biosynthetic pathways.

\section{Conclusion}

Four crude polysaccharides (termed CIOPG, CIOPF, CIOPL, and CIOPGL) and four derived neutral polysaccharides (NIOPG, NIOPF, NIOPL, and NIOPGL) were extracted and purified from mycelia of I. obliquus generated by liquid fermentation using respective carbon sources Glc, Fru, Lac, and Glc+Lac. Structural analysis by FTIR, GC-MS, and several chemical methods revealed considerable variation in MW, monosaccharide composition, and glycosidic linkages of the four NIOPs. In vitro experiments showed that proliferation of macrophage cells (RAW264.7) and tumor cells (HeLa and S180) differed greatly among groups treated with the various CIOPs and NIOPs. qRT-PCR analysis revealed that expression levels of pgi and uge, two key genes involved in polysaccharide synthetic pathway, differed significantly depending on carbon source used. Carbon source clearly affected synthesis, structure, and function of fungal polysaccharides, based on differential regulation of expression levels of genes involved in polysaccharide biosynthesis.

\section{Acknowledgments}

This study was supported by the Natural Science Foundation of Hubei Province, China (Grant No. 2020CFB527), Key Research and Development Program of Hubei Province, China (Grant No. 2020BAB095) and Fundamental Research Funds for the Central Universities of China (Grant No. 2662019PY066). The authors are grateful to Dr. S. Anderson for English editing of the manuscript.

\section{Conflict of Interest}

The authors have no financial conflicts of interest to declare.

\section{References}

1. Wei ZH, Chen N, Li YJ, Fan QL, Yu T-F, Wang K-X, et al. 2018. Glucose fed-batch integrated dissolved oxygen control strategy enhanced polysaccharide, total triterpenoids and inotodiol production in fermentation of a newly isolated Inonotus obliquus strain. Process Biochem. 66: 1-6.

2. Wold CW, Kjeldsen C, Corthay A, Rise F, Christensen BE, Duus JØ, et al. 2018. Structural characterization of bioactive heteropolysaccharides from the medicinal fungus Inonotus obliquus (Chaga). Carbohydr. Polym. 185: 27-40.

3. Lee KH, Kim H, Oh SH, Hwang JH, Yu KW. 2017. Immunomodulating Activity of crude polysaccharide from Inonotus obliquus sclerotia by fractionation including MeOH reflux. Korean J. Food Nutr. 30: 96-104.

4. Kim YO, Park HW, Kim JH, Lee JY, Moon SH, Shin CS. 2006. Anti-cancer effect and structural characterization of endopolysaccharide from cultivated mycelia of Inonotus obliquus. Life Sci. 79: 72-80.

5. Wang C, Li W, Chen Z, Gao X, Yuan G, Pan Y, et al. 2018. Effects of simulated gastrointestinal digestion in vitro on the chemical properties, antioxidant activity, $a$-amylase and a-glucosidase inhibitory activity of polysaccharides from Inonotus obliquus. Food Res. Int. 103: 280-288.

6. Zhang CJ, Guo JY, Cheng H, Li L, Liu Y, Shi Y, et al. 2020. Spatial structure and anti-fatigue of polysaccharide from Inonotus obliquus. Int. J. Biol. Macromol. 151: 855-860.

7. Liu P, Xue J, Tong SS, Dong WX, Wu PP. 2018. Characterization and hypoglycaemic activities of two polysaccharides from Inonotus obliquus. Molecules 23:1948. 
8. Chen HJ, Chen YS, Liu SL, Liou BK, Chen CS. 2020. The influence of submerged fermentation of Inonotus obliquus with control atmosphere treatment on enhancing bioactive ingredient contents. Appl. Biochem. Biotechnol. 191: 412-425.

9. Wang MY, Zhao ZZ, Zhou X, Hu JR, Xue J, Liu X, et al. 2019. Simultaneous use of stimulatory agents to enhance the production and hypoglycaemic activity of polysaccharides from Inonotus obliquus by submerged fermentation. Molecules 24: 4400.

10. Li H, Wu J, Chen Q. 2019. Effect of farnesol, a Kind of quorum sensing molecule on the production of triterpenoids and betulinic acid in the submerged fermentation of Inonotus obliquus. J. Chin. Inst. Food Sci. Technol. 19: 171-176.

11. Xu X, Quan L, Shen M. 2015. Effect of chemicals on production, composition and antioxidant activity of polysaccharides of Inonotus obliquus. Int. J. Biol. Macromol. 77: 143-150.

12. Dubois M, Gilles KA, Hamilton JK, F. S. 1956. Colorimetric method for determiation of sugars and related substances. Anal. Chem. . 28: 350-356.

13. Kruger NJ. 1994. The Bradford Method for Protein Quantitation. In: Walker J.M. (eds) Basic Protein and Peptide Protocols. Methods in Molecular Biology, vol. 32. Humana Press.

14. Wang T, He H, Liu X, Liu C, Liang Y, Mei Y. 2019. Mycelial polysaccharides of Lentinus edodes (shiitake mushroom) in submerged culture exert immunoenhancing effect on macrophage cells via MAPK pathway. Int. J. Biol. Macromol. 130: 745-754.

15. Zhang J, Mei Z, Huang X, Ding Y, Liang Y, Mei Y. 2020. Inhibition of Maillard reaction in production of low-molecular-weight chitosan by enzymatic hydrolysis. Carbohydr. Polym. 236: 116059.

16. Zhang Y, Wang Z, Li D, Zang W, Zhu H, Wu P, et al. 2018. A polysaccharide from Antrodia cinnamomea mycelia exerts antitumor activity through blocking of TOP1/TDP1-mediated DNA repair pathway. Int. J. Biol. Macromol. 120: 1551-1560.

17. Kong F, Cao M, Sun P, Liu W, Mao Y. 2015. Selection of reference genes for gene expression normalization in Pyropia yezoensis using quantitative real-time PCR. J. Appl. Phycol. 27: 1003-1010.

18. Chang CJ, Lin CS, Lu CC, Martel J, Ko YF, Ojcius DM, et al. 2015. Ganoderma lucidum reduces obesity in mice by modulating the composition of the gut microbiota. Nat. Commun. 6: 7489 .

19. Mei YX, Chen HX, Zhang J, Zhang XD, Liang YX. 2013. Protective effect of chitooligosaccharides against cyclophosphamideinduced immunosuppression in mice. Int. J. Biol. Macromol. 62: 330-335.

20. Tang YJ, Zhu LL, Li DS, Mi ZY, Li HM. 2008. Significance of inoculation density and carbon source on the mycelial growth and Tuber polysaccharides production by submerged fermentation of Chinese truffle Tuber sinense. Process Biochem. 43: 576-586.

21. West TP. 2011. Effect of carbon source on polysaccharide production by alginate-entrapped Aureobasidium pullulans ATCC 42023 cells. J. Basic Microb. 51: 673-677.

22. Ding Z, Jia SR, Han PP, Yuan NN, Tan N. 2013. Effects of carbon sources on growth and extracellular polysaccharide production of Nostoc flagelliforme under heterotrophic high-cell-density fed-batch cultures. J. Appl. Phycol. 25: 1017-1021.

23. Wasser SP, Elisashvili VI, Tan K-K. 2003. Effects of carbon and nitrogen sources in the medium on Tremella mesenterica Retz.:Fr. (Heterobasidiomycetes) growth and polysaccharide production. Int. J. Med. Mushrooms 5: 49-56.

24. Hwang HJ, Kim SW, Choi JW, Yun JW. 2003. Production and characterization of exopolysaccharides from submerged culture of Phellinus linteus KCTC 6190. Enzyme Microb. Technol. 33: 309-319.

25. Troxler LJ, Werren JP, Schaffner TO, Mostacci N, Vermathen P, Vermathen M, et al. 2019. Carbon source regulates polysaccharide capsule biosynthesis in Streptococcus pneumoniae. J. Biol. Chem. 294: 17224-17238.

26. Xu L, Lu Y, Cong Y, Zhang P, Han J, Song G, et al. 2019. Polysaccharide produced by Bacillus subtilis using burdock oligofructose as carbon source. Carbohydr. Polym. 206: 811-819.

27. Peng L, Li J, Liu Y, Xu ZH, Wu JY, Ding ZY, et al. 2016. Effects of mixed carbon sources on galactose and mannose content of exopolysaccharides and related enzyme activities in Ganoderma lucidum. RSC Adv. 6: 39284-39291.

28. Han PP, Yao SY, Guo RJ, Shen SG, Yan RR, Tan ZL, et al. 2017. The relationship between monosaccharide composition of extracellular polysaccharide and activities of related enzymes in Nostoc flagelliforme under different culture conditions. Carbohydr. Polym. 174: 111-119.

29. Kim SK, Lee CG, Yun HS. 2003. Heavy metal adsorption characteristics of extracellular polysaccharide produced by Zoogloea ramigera grown on various carbon sources. J. Microbiol. Biotechnol. 13: 745-750.

30. Wang Q, Wang F, Xu Z, Ding Z. 2017. Bioactive mushroom polysaccharides: a review on monosaccharide composition, biosynthesis and regulation. Molecules 22: 955

31. Zhu ZY, Liu XC, Dong FY, Guo MZ, Wang XT, Wang Z, et al. 2016. Influence of fermentation conditions on polysaccharide production and the activities of enzymes involved in the polysaccharide synthesis of Cordyceps militaris. Appl. Microbiol. Biotechnol. 100: 3909-3921.

32. Wei ZH, Liu L, Guo XF, Li YJ, Hou BC, Fan QL, et al. 2016. Sucrose fed-batch strategy enhanced biomass, polysaccharide, and ganoderic acids production in fermentation of Ganoderma lucidum 5.26. Bioprocess Biosyst. Eng. 39: 37-44.

33. Peng L, Qiao SK, Xu ZH, Guan F, Ding ZY, Gu ZH, et al. 2015. Effects of culture conditions on monosaccharide composition of Ganoderma lucidum exopolysaccharide and on activities of related enzymes. Carbohydr. Polym. 133: 104-109. 